\title{
A general framework for the optimal approximation of circular arcs by parametric polynomial curves
}

\author{
Aleš Vavpetič ${ }^{\mathrm{a}, \mathrm{b}, *}$, Emil Žagar ${ }^{\mathrm{a}, \mathrm{b}, *}$ \\ ${ }^{a}$ Faculty of Mathematics and Physics, University of Ljubljana, Jadranska 19, Ljubljana, \\ Slovenia \\ ${ }^{b}$ Institute of Mathematics, Physics and Mechanics, Jadranska 19, Ljubljana, Slovenia
}

\begin{abstract}
We propose a general framework for a geometric approximation of circular arcs by parametric polynomial curves. The approach is based on a constrained uniform approximation of an error function by scalar polynomials. The system of nonlinear equations for the unknown control points of the approximating polynomial given in the Bézier form is derived and a detailed analysis provided for some low degree cases which were not studied yet. At least for these cases the solutions can be, in principal, written in a closed form, and provide the best known approximants according to the simplified radial distance. A general conjecture on the optimality of the solution is stated and several numerical examples conforming theoretical results are given.
\end{abstract}

Key words: geometric interpolation, circular arc, parametic polynomial, Bézier curve, optimal approximation

2010 MSC: 65D05, 65D07, 65D17

\section{Introduction}

Circular arcs are one of the fundamental geometric primitives and together with straight lines they have been one of the cornerstones of several graphical and control systems. Later on parametric polynomial representations of geometric objects have been widely used in applications and success-

\footnotetext{
${ }^{*}$ Corresponding author

Email addresses: ales.vavpetic@fmf.uni-lj.si (Aleš Vavpetič), emil.zagar@fmf.uni-lj.si (Emil Žagar)

Preprint submitted to Journal of Computational and Applied Mathematics June 6, 2018
} 
fully upgraded to non-uniform rational basis splines (NURBS) which nowadays provide an intuitive approach towards to the construction and modelling of curves and surfaces used in computer aided geometric design (CAGD) and related fields. However, there is still an interest in parametric polynomial curves, since they provide even more simple representations of geometric objects and might still be in use in some software standards. On the other hand, optimal approximation of special classes of functions or parametric objects by polynomials has always been a theoretical issue (Chebyshev alternation theorem [1], Stone-Weierstrass approximation theorem [2], etc.). Circular arcs form one such class of curves, since it is well known that a circular arc of positive length can not be exactly represented in a polynomial form.

A common way to construct parametric polynomial approximants of a circular arc is to interpolate corresponding geometric quantities. This usually include interpolation of boundary points, corresponding tangent directions, signed curvatures, etc. The result are so called geometric parametric polynomial approximants ( $G^{k}$ approximants), which can be put together to geometrically smooth spline curves.

When we are dealing with approximations, the fundamental question is a measure of a distance between a parametric polynomial approximant and a circular arc. One of the standard measures in this case is the radial distance measuring the distance of the point on the parametric polynomial to the corresponding point on the circular arc in the radial direction. It can be shown that under some additional assumptions it coincides with the well known Hausdorff distance $([3,4])$. It is more common to use a simplified version of the radial distance, the difference between the square of the distance of the point on the parametric polynomial curve to the center of the circular arc and the square of its radius. The later one is more attractive since it simplifies the analysis of the existence and uniqueness of the approximant but still preserves the optimality of the approximation order. However, it is important to emphasize that the optimal solutions according to this two measures do not coincide in general.

The list of literature dealing with different types of geometric approximants of a circular arc is long and we shall mention just the most relevant references according to our approach described later. Parabolic $G^{0}$ interpolants were considered in [5]. This is actually one of only a few cases where the optimality of the solution was proved. Different types of $G^{1}$ and $G^{2}$ cubic geometric interpolants were given in early papers [6] and [7]. Several types of quartic and quintic Bézier curves were considered in [3], and deeper 
analysis of some geometric quintic approximants can be found in [8]. Many new cubic and quartic approximants were also proposed in $[9,10,11,12,13]$. However, in none of the above papers the optimality of the solution has been considered. The paper [14] is beside [5] the only one where optimality of some approximants was formally shown. The authors managed to prove it for cubic $G^{1}$ and quartic $G^{2}$ approximants.

Some authors also considered the approximation of circular arcs by general degree parametric polynomials. In [15], the Taylor type geometric interpolation, i.e., interpolation at just one point was considered for all odd degree polynomials. For even degree ones the results can be found in [16] and in a more general form in [17]. The approximation of the whole circle by Lagrange type approximants can be found in [18] and in [4].

The aim of this paper is to present a general framework providing optimal geometric approximants for general degree $n$ of the parametric polynomial and for any order $k$ of geometric smoothness. The idea relies on the constrained uniform approximation of the error function by scalar polynomials.

The paper is organized as follows. In Section 2 the problem is explained in detail and the radial distance and its simplification are precisely introduced. A general conjecture that the proposed approach provides optimal solutions is stated. Section 3 concerns a constrained uniform approximation of an error function by scalar polynomials. A general theory is briefly revised and some particular cases needed later are carefully analysed. Next section describes optimal $G^{k}$ approximation of circular arcs by parametric polynomial curves. In particular, it provides the system of nonlinear equations which has to be solved. In Section 5 some particular cases are studied in detail. For some of them the optimality is reconfirmed, but for all of them it is shown that they provide the minimal simplified radial distance among all known approximants. In the last section some concluding remarks and suggestions for possible future research are given.

\section{Preliminaries}

We shall consider the following problem. Let $c:[-\varphi, \varphi] \rightarrow \mathbb{R}^{2}, 0<\varphi \leq$ $\pi / 2$ be a standard nonpolynomial parameterization of a circular arc. Due to simple affine transformations it is enough to consider the unit circular arcs only, centred at the origin and symmetric with respect to the first coordinate axis. Thus we can assume that $\boldsymbol{c}(s)=(\cos s, \sin s)^{T}$. Our goal is to find as good as possible approximation of $\boldsymbol{c}$ by parametric polynomial curve $\boldsymbol{p}_{n}$ : 
$[-1,1] \rightarrow \mathbb{R}^{2}$ of degree $n \in \mathbb{N}$. It is convenient to express $\boldsymbol{p}_{n}=\left(x_{n}, y_{n}\right)^{T}$, where $x_{n}$ and $y_{n}$ are polynomials of degree at most $n$, in Bézier form, i.e.,

$$
\boldsymbol{p}_{n}(t)=\sum_{j=0}^{n} B_{j}^{n}(t) \boldsymbol{b}_{j}
$$

where $B_{j}^{n}, j=0,1, \ldots, n$, are (reparameterized) Bernstein polynomials over $[-1,1]$, given as

$$
B_{j}^{n}(t)=\left(\begin{array}{c}
n \\
j
\end{array}\right)\left(\frac{1+t}{2}\right)^{j}\left(\frac{1-t}{2}\right)^{n-j},
$$

and $\boldsymbol{b}_{j} \in \mathbb{R}^{2}, j=0,1, \ldots, n$, are the control points.

The quality of the approximation will be measured by radial distance. For each point on the parametric curve $\boldsymbol{p}_{n}$ the closest point on the circular arc $\boldsymbol{c}$ in the radial direction will be considered. In general, it might happen that no such point exists on $\boldsymbol{c}$, but some further restrictions on $\boldsymbol{p}_{n}$ will override this problem. The formal definition of the radial distance $\widetilde{\psi}_{n}$ is

$$
\widetilde{\psi}_{n}:[-1,1] \rightarrow[0, \infty), \quad \widetilde{\psi}_{n}(t):=\left|\sqrt{x_{n}(t)^{2}+y_{n}(t)^{2}}-1\right|=\left|\left\|\boldsymbol{p}_{n}(t)\right\|_{2}-1\right|,
$$

where $\|\cdot\|_{2}$ is the standard Euclidean norm on $\mathbb{R}^{2}$. Function $\widetilde{\psi}_{n}$ is an upper bound for the parametric distance $d_{P}$, studied in detail in [15]. For $\boldsymbol{c}$ and $\boldsymbol{p}_{n}$ it is defined as

$$
d_{P}\left(\boldsymbol{c}, \boldsymbol{p}_{n}\right)=\inf _{\rho} \max _{t \in[-1,1]}\left\|(\boldsymbol{c} \circ \rho)(t)-\boldsymbol{p}_{n}(t)\right\|_{2},
$$

where $\rho:[-1,1] \rightarrow[-\varphi, \varphi]$ is a smooth bijection for which $\rho^{\prime}>0$. Clearly, $d_{P}$ is in general an upper bound for the well known Hausdorff distance $d_{H}$. If the radial distance between $\boldsymbol{c}$ and $\boldsymbol{p}_{n}$ is well defined, it can be shown that actually

$$
d_{H}(\boldsymbol{c}, \boldsymbol{b})=d_{P}\left(\boldsymbol{c}, \boldsymbol{p}_{n}\right)=\max _{t \in[-1,1]} \widetilde{\psi}_{n}(t)
$$

(see [3] or [4] for details). Due to computational reasons it is easier to consider a simplified (signed) radial error

$$
\psi_{n}:[-1,1] \rightarrow[0, \infty), \quad \psi_{n}(t):=x_{n}(t)^{2}+y_{n}(t)^{2}-1=\left\|\boldsymbol{p}_{n}(t)\right\|_{2}^{2}-1,
$$


since no irrational functions are involved but the location of zeros and extrema remains the same as for $\widetilde{\psi}_{n}$. The approximation of circular arc $\boldsymbol{c}$ by parametric polynomial $\boldsymbol{p}_{n}$ now reduces to the study of the optimality of $\psi_{n}$.

In practice, some additional properties of $\boldsymbol{p}_{n}$ are required, such as interpolation of boundary points, tangent directions,... More precisely, some geometric interpolation conditions are prescribed at the boundary. These are given in the following definition.

Definition 1. A circular arc $\boldsymbol{c}$ and a parametric polynomial curve $\boldsymbol{p}_{n}$ share a geometric contact of order $k \in \mathbb{N}$ at the boundary points $\boldsymbol{c}( \pm \varphi)$, if there exists a smooth regular bijective reparameterization $\rho:[-1,1] \rightarrow[-\varphi, \varphi]$ with $\rho^{\prime}>0$, such that

$$
\frac{d^{j} \boldsymbol{p}_{n}}{d t^{j}}( \pm 1)=\frac{d^{j}(\boldsymbol{c} \circ \rho)}{d t^{j}}( \pm 1), \quad j=0,1, \ldots, k .
$$

We say that $\boldsymbol{p}_{n}$ is a $G^{k}$ approximation of $\boldsymbol{c}$ in this case.

The following important result characterizes $G^{k}$ approximants of circular arcs.

Lemma 2. A parametric polynomial $\boldsymbol{p}_{n}$ is a $G^{k}$ approximation of the circular arc $\boldsymbol{c}$ if and only if $\psi_{n}$ has zeros of multiplicity $k+1$ at $t= \pm 1$.

The proof of this lemma can be found in [3]. It is well known that parametric polynomials can not reproduce circular arcs of positive length. So for a $G^{k}$ approximat $\boldsymbol{p}_{n}$ of the circular arc $\boldsymbol{c}$ it follows from Lemma 2 that

$$
\psi_{n}(t)=\psi_{n, k}(t):=C p_{2 n, k}(t)
$$

where $C \in \mathbb{R}$ is a nonzero constant and

$$
p_{2 n, k}(t):=\left(1-t^{2}\right)^{k+1} q_{n, k}(t)
$$

is a polynomial of degree $2 n$ with $q_{n, k}$ being monic of degree $2 n-2 k-2$. By (1) and (2), $C$ and $p_{2 n, k}$ both depend on control points $\boldsymbol{b}_{j}, j=0,1, \ldots, n$, which further have to fulfil some additional constraints, ensuring the $G^{k}$ continuity from Definition 1. In order to find the best approximant according to (2), the nonlinear optimization problem have to be solved. If we write $C=C\left(\boldsymbol{b}_{0}, \ldots, \boldsymbol{b}_{n}\right)$ and $p_{2 n, k}(t)=p_{2 n, k}\left(t ; \boldsymbol{b}_{0}, \ldots, \boldsymbol{b}_{n}\right)$, then we are looking for

$$
\min _{\boldsymbol{b}_{0}, \ldots, \boldsymbol{b}_{n}} \max _{t \in[-1,1]}\left|C\left(\boldsymbol{b}_{0}, \ldots, \boldsymbol{b}_{n}\right) p_{2 n, k}\left(t ; \boldsymbol{b}_{0}, \ldots, \boldsymbol{b}_{n}\right)\right| \text {. }
$$


This is definitely very hard nonlinear constrained optimization task. The authors in several papers simplified it in a way that they have chosen a polynomial $q_{n, k}$ from (4) in advance and then minimized the constant $C$. This can be done, e.g., by prescribing zeros of $q_{n, k}$. However, the quality of the approximant heavily relies on the selection of zeros and optimality is not guaranteed. The only known direct optimizations (5) seem to be in [5] and in [14], where the authors considered an optimal quadratic $G^{0}$, cubic $G^{1}$ and quartic $G^{2}$ approximation of circular arcs. All these problems were dealing with one parametric families of approximants, and it seems that there are no results known about optimal approximants when several parameters are involved.

Here we propose a new general framework which might provide optimal approximants in any case. We again choose $q_{n, k}$ of degree $2 n-2 k-2$, but now in a way that it provides a minimum of

$$
\left\|p_{2 n, k}\right\|=\max _{t \in[-1,1]}\left|\left(1-t^{2}\right)^{k+1} q_{n, k}(t)\right| .
$$

The polynomial $p_{2 n, k}$, which minimizes (6), will be denoted by $p_{2 n, k}^{*}$, and the corresponding $q_{n, k}$ by $q_{n, k}^{*}$. The idea comes from the constrained uniform approximation of the zero function on $[-1,1]$ by monic polynomials and will be considered in detail in the next section. However, this choice does not a priori guarantee the optimality of the approximant as one might quickly conclude from uniform polynomial approximation of functions. There might exist approximants which do not provide minimal $\left\|p_{2 n, k}\right\|$, but they provide a constant $C$ small enough that corresponding $\left|\psi_{n, k}\right|$ would be smaller than the one arising from $p_{2 n, k}^{*}$. However, results of the present paper show that there is some hope that this actually can not happen.

It is clear that $p_{2 n, k}^{*}$, which minimizes (6), does not depend on $\boldsymbol{b}_{j}, j=$ $0,1, \ldots, n$. It depends only on $n, k$, and the properties of the norm (6). Once $p_{2 n, k}^{*}$ is determined, then control points $\boldsymbol{b}_{j}, j=0,1, \ldots, n$, are given as a solution of the system of nonlinear equations, and we are left with the minimization of $C\left(\boldsymbol{b}_{0}, \ldots, \boldsymbol{b}_{n}\right)$ (i.e., we have to choose a solution providing minimal $\left.\left|C\left(\boldsymbol{b}_{0}, \ldots, \boldsymbol{b}_{n}\right)\right|\right)$. The main purpose of this paper is to show that the proposed approach reproduces the above mentioned optimal solutions obtained in [5] and in [14] and provides new solutions for $G^{0}$ cubic and $G^{1}$ quartic approximants possessing the smallest known error. This leads us to the following conjecture. 
Conjecture 3. The best $G^{k}$ geometric approximant $\boldsymbol{p}_{n}$ of the circular arc $\boldsymbol{c}$ according to the error measure $\psi_{n, k}$, given by (3), arises from the choice $p_{2 n, k}^{*}$ determining $\boldsymbol{b}_{j}, j=0,1, \ldots, n$, with minimal $\left|C\left(\boldsymbol{b}_{0}, \ldots, \boldsymbol{b}_{n}\right)\right|$.

In the following section the general approach to the construction of $p_{2 n, k}^{*}$ will be described.

\section{Constrained uniform approximation}

In this section the optimal approximation of the zero function by polynomials of the form $p_{2 n, k}(t)=\left(1-t^{2}\right)^{k+1} q_{n, k}(t)$, where $q_{n, k}$ is a monic polynomial of degree $2 n-2 k-2$, will be considered. In particular, we shall study the following problem: For any $k, n \in \mathbb{N}$, such that $0 \leq k<n$, find a monic polynomial $q_{n, k}^{*}$ of degree $2 n-2 k-2$ for which $p_{2 n, k}^{*}(t)=\left(1-t^{2}\right)^{k+1} q_{n, k}^{*}(t)$ provides the minimal max norm on $[-1,1]$.

Suppose that $q_{n, k}:[-1,1] \rightarrow \mathbb{R}$ is a monic polynomial of degree $2 n-2 k-2$. Let us define the polynomial $p_{2 n, k}$ of degree $2 n$ by

$$
p_{2 n, k}(t)=\left(1-t^{2}\right)^{k+1} q_{n, k}(t) .
$$

It follows from [19] that there exists the unique monic polynomial $q_{n, k}^{*}$ of degree $2 n-2 k-2$, such that $p_{2 n, k}^{*}(t)=\left(1-t^{2}\right)^{k+1} q_{n, k}^{*}(t)$ has the minimal max norm over all polynomials of the form (7). The polynomial $p_{2 n, k}^{*}$ is characterized by the following property [19, Theorem 3.1]: There exist $2 n-$ $2 k-1$ points $-1<a_{0}<\ldots<a_{2 n-2 k-2}<1$ such that $\left\|p_{2 n, k}^{*}\right\|=\left|p_{2 n, k}^{*}\left(a_{0}\right)\right|$ and $p_{2 n, k}^{*}\left(a_{0}\right)=(-1)^{i} p_{2 n, k}^{*}\left(a_{i}\right)$ for $i=1, \ldots, 2 n-2 k-2$. Since we are dealing with polynomials defined over the symmetric interval $[-1,1]$, and since for every monic polynomial $r$ of even degree also $t \mapsto \frac{1}{2}(r(t)+r(-t))$ is a monic of norm no greater than $\|r\|$, the polynomials $q_{n, k}^{*}$ and $p_{2 n, k}^{*}$ must be even. Hence

$$
p_{2 n, k}^{*}(t)=\left(1-t^{2}\right)^{k+1}\left(t^{2}-t_{1}^{2}\right) \cdots\left(t^{2}-t_{n-k-1}^{2}\right)
$$

for some $0<t_{1}<\ldots<t_{n-k-1}<1$. Some special cases which can be analyzed analytically and will be needed later for the construction of particular geometric approximants, will now be considered in detail.

\subsection{The case $k=0$}

The polynomial $p_{2 n, 0}^{*}$ has exactly $2 n$ single roots. Two of them are on the boundary of the interval. From a general theory of uniform approximation by 
polynomials it follows that $p_{2 n, 0}^{*}$ is a scaled and dilated Chebyshev polynomial $T_{2 n}$ of degree $2 n$, more precisely

$$
p_{2 n, 0}^{*}(t)=-\frac{2^{1-2 n}}{\cos ^{2 n}\left(\frac{\pi}{4 n}\right)} T_{2 n}\left(\cos \left(\frac{\pi}{4 n}\right) t\right) .
$$

It is easy to deduce $q_{n, 0}^{*}$ since its zeros must be precisely the $2 n-2$ interior zeros of $p_{2 n, 0}^{*}$. In particular, if $n=2$, we have $t_{1}=\sqrt{3-2 \sqrt{2}}$, and for $n=3$ the pozitive zeros are $t_{1}=2-\sqrt{3}$ and $t_{2}=\sqrt{3}-1$.

\subsection{The case $k=n-3$}

By (8), the polynomial $p_{2 n, n-3}^{*}$ has zeros of multiplicity $n-2$ at $t= \pm 1$. Additionally, it has four simple zeros on $(-1,1)$ and a local extremum at $t=0$ due to the symmetry. Consequently, its derivative has zeros of multiplicity $n-3$ at $t= \pm 1$, a simple zero at $t=0$ and four additional symmetric simple zeros on $(-1,1)$. Since the signs of the leading coefficients of $p_{2 n, n-3}^{*}$ and its derivative are the same, the derivative can be written as

$$
\frac{d p_{2 n, n-3}^{*}}{d t}(t)=-2 n\left(1-t^{2}\right)^{n-3} t\left(t^{2}-(1-a)\right)\left(t^{2}-(1-b)\right), \quad 0<b<a<1
$$

Integration of the previous form gives

$$
p_{2 n, n-3}^{*}(t)=\left(1-t^{2}\right)^{n-2}\left(\left(1-t^{2}\right)^{2}-\frac{n}{n-1}(a+b)\left(1-t^{2}\right)+\frac{n}{n-2} a b\right) .
$$

Since the extrema of $p_{2 n, n-3}^{*}$ are $\pm \sqrt{1-a}, \pm \sqrt{1-b}$, and 0 , characterization of the best approximat implies

$$
p_{2 n, n-3}^{*}(0)=-p_{2 n, n-3}^{*}(\sqrt{1-a})=p_{2 n, n-3}^{*}(\sqrt{1-b}) .
$$

The last equality in (10) leads to

$$
-a^{n-1}\left(\frac{n}{(n-1)(n-2)} b-\frac{1}{n-1} a\right)=b^{n-1}\left(\frac{n}{(n-1)(n-2)} a-\frac{1}{n-1} b\right) .
$$

Multiplication by $\frac{n-1}{a^{n}}$ gives

$$
\zeta(\lambda):=\lambda^{n}-\frac{n}{n-2} \lambda^{n-1}-\frac{n}{n-2} \lambda+1=0,
$$


where $\lambda:=\frac{b}{a} \in(0,1)$. Since $\zeta(0)=1>0$ and $\zeta(1)=-4 /(n-2), \zeta$ must have at least one root on $(0,1)$. But then by the Descartes rule of signs there must be exactly two positive roots. Due to the symmetry, roots of $\zeta$ must appear in pairs $\lambda, 1 / \lambda$. Consequently we have the unique root on $(0,1)$. In particular, if $n=4$, we have $\zeta(\lambda)=\lambda^{4}-2 \lambda^{3}-2 \lambda+1=0$ and

$$
\lambda=(\sqrt{3}-\sqrt{2} \sqrt[4]{3}+1) / 2 .
$$

Since $b=\lambda a$, the first equality in (10) and the equation (11) imply an equation for the unknown $a$, namely

$$
\left(a-\frac{1}{\lambda}\right)^{2}\left(\lambda^{2}(2 \lambda-1) a^{2}+2 \lambda(2 \lambda-1) a+6 \lambda^{3}+6 \lambda-3\right)=0 .
$$

However, $\lambda<1$, and $a$ must be the unique positive zero of the second factor in (13). Some calculations reveal that $a$ can be written as

$$
a=\sqrt{1+\sqrt{3}+\sqrt{24+14 \sqrt{3}}}-\frac{1}{2}(1+\sqrt{3}+\sqrt{2} \sqrt[4]{3}) .
$$

The polynomial $p_{8,1}^{*}$ then reads as

$$
p_{8,1}^{*}(t)=-\left(1-t^{2}\right)^{2}\left(t^{4}+\frac{2}{3}(2(\lambda+1) a-3) t^{2}+\frac{1}{3}\left(3-4(\lambda+1) a+6 \lambda a^{2}\right)\right),
$$

where $\lambda$ is given by (12) and $a$ by (14). Two positive zeros $t_{1,2}$ of $p_{8,1}^{*}$ can be found as a solution of the quadratic equation arising from the quartic factor in $(15)$.

\subsection{The case $k=n-2$}

Similar calculations as in the previous case can be done for $k=n-2$. It can be shown that the polynomial $p_{2 n, n-2}^{*}$ must be of the form

$$
p_{n, n-2}^{*}(t)=\left(1-t^{2}\right)^{n-1}\left(t^{2}-1+\frac{n}{n-1} a\right),
$$

where $a$ is the unique solution of

$$
a^{n}+n a-(n-1)=0
$$

on $(0,1)$. In particular, for $n=3$ we have $a=\sqrt[3]{\sqrt{2}+1}-\sqrt[3]{\sqrt{2}-1}$ and

$$
p_{6,1}^{*}(t)=\left(1-t^{2}\right)^{2}\left(t^{2}-1+\frac{3}{2} a\right) \text {. }
$$

Thus $t_{1}=\sqrt{1-\frac{3}{2} a}$. 


\section{Optimal $G^{k}$ approximation of circular arcs}

In previous section constrained uniform minimization by polynomials was studied in detail. In order to use the obtained results, let us consider a general problem of $G^{k}$ approximation of circular arcs by parametric polynomials of arbitrary degree $n$. Suppose that the approximant $\boldsymbol{p}_{n}$ is given by (1). Quite clearly, $\boldsymbol{p}_{n}$ has $2 n+2$ free parameters, i.e., the coordinates of the control points $\boldsymbol{b}_{j}, j=0,1, \ldots, n$. Since the circular arc $\boldsymbol{c}$ is symmetric with respect to abscissa, so is the approximant $\boldsymbol{p}_{n}$. Consequently, its control points must be symmetric too, and the number of free parameters reduces to $n+1$. Additionally, $G^{0}$ condition at a particular point prescribes two parameters, and each $G^{\ell}$ condition, $1 \leq \ell \leq k$, reduces the number of free parameters by one ([21]). Finally, $G^{k}$ approximant is determined by $n+1-2-k=n-k-1$ parameters. Particularly, if $k=0$, the first and the last control points must be $\boldsymbol{b}_{0}=(\cos \varphi,-\sin \varphi)^{T}$ and $\boldsymbol{b}_{n}=(\cos \varphi, \sin \varphi)^{T}$. If $k=1$, additionally $\boldsymbol{b}_{1}=\boldsymbol{b}_{0}+d \boldsymbol{c}^{\prime}(-\varphi)$ and $\boldsymbol{b}_{n-1}=\boldsymbol{b}_{n}-d \boldsymbol{c}^{\prime}(\varphi)$ for some $d>0$. Some similar, but more complicated relations can be derived for $k \geq 2$, too.

Since we are interested in the optimal $G^{k}$ approximation, we shall follow our proposed approach and choose

$$
\psi_{n, k}(t)=\left\|\boldsymbol{p}_{n}(t)\right\|_{2}^{2}-1=C p_{2 n, k}^{*}(t)=C\left(1-t^{2}\right)^{k+1} q_{n, k}^{*}(t),
$$

where $p_{2 n, k}^{*}$ minimizes (6). By (8), $q_{n, k}^{*}$ has $2 n-2 k-2$ symmetric roots on $(-1,1)$. Let $0<t_{1}<t_{2}<\cdots<t_{n-k-1}<1$ be the positive ones. Then

$$
\psi_{n, k}\left(t_{i}\right)=\left\|\boldsymbol{p}_{n}\left(t_{i}\right)\right\|_{2}^{2}-1=0, \quad i=1,2, \ldots, n-k-1,
$$

is a system of $n-k-1$ nonlinear equations for the $n-1-k$ unknown parameters determining the approximant $\boldsymbol{p}_{n}$. It might have several solutions, and we are interested in that one which minimizes the absolute value of $C$ in (17). Since due to the symmetry $\psi_{n, k}$ must have an extreme point at 0 , and all the extrema are by construction of the same magnitude, we have

$$
C=\frac{\left\|\boldsymbol{p}_{n}(0)\right\|_{2}^{2}-1}{q_{n, k}^{*}(0)} .
$$

Among all possible solutions of (18), we thus choose the one providing $\boldsymbol{p}_{n}$ for which (19) has the minimal absolute value.

There is a little hope that the problem can be solved for general $k$ and $n$. Thus we will concentrate on some specific low degree cases and we shall 
confirm that our approach either reproduces the best solution or implies the solution for which the error is the smallest among all by now known approximants. This gives some hope that the Conjecture 3 might hold true for any $k$ and $n$.

\section{Some particular cases}

The first nontrivial case is $n=2$ and $k=0$, i.e., quadratic approximation via interpolation of boundary points of the circular arc $\boldsymbol{c}$. This case has already been considered in [5] and the best solution was characterized. We reconsidered it by our approach using the results from Subsection 3.1 and confirmed the result. In addition, we also derived an asymptotic expansion of $C$, which was not provided in [5], namely $C=-\varphi^{4} / 4+\mathcal{O}\left(\varphi^{5}\right)$ and consequently

$$
\max _{t \in[-1,1]}\left|\psi_{2,0}(t)\right|=\frac{3-2 \sqrt{2}}{4} \varphi^{4}+\mathcal{O}\left(\varphi^{5}\right) \approx 0.0429 \varphi^{4}+\mathcal{O}\left(\varphi^{5}\right) .
$$

The case $n=2$ and $k=1$ is not interesting, since the inner control point $\boldsymbol{b}_{1}$ is uniquely determined by $G^{1}$ condition as the intersection of tangent lines to the boundary points of the circular arc. Thus, there is nothing to be optimized.

Let us consider the case $n=3$ and $k=0$ now. It was partially considered in [7]. The author only reported that it leads to the solution of the nonlinear

biquadratic system, but no proof of the existence and uniqueness of the solution was provided. Here we fill this gap by the formal proof arising from our approach based again on the results from Subsection 3.1. The ideas in the proof will be also used for the analysis of the $G^{1}$ quartic case, the main contribution of our discussion.

Due to $G^{0}$ conditions, the control points of the approximant $\boldsymbol{p}_{3}$ must be

$$
\boldsymbol{b}_{0}=(\cos \varphi,-\sin \varphi)^{T}, \quad \boldsymbol{b}_{1}=(\xi,-\eta)^{T}, \quad \boldsymbol{b}_{2}=(\xi, \eta)^{T}, \quad \boldsymbol{b}_{3}=(\cos \varphi, \sin \varphi)^{T},
$$

where obviously $\xi>1$ and $\eta>0$. By (9)

$$
p_{6,0}(t)=-\frac{1}{32 \cos \left(\frac{\pi}{12}\right)^{6}} T_{6}\left(\cos \left(\frac{\pi}{12}\right) t\right),
$$

and its positive zeros on $(0,1)$ are $t_{1}=2-\sqrt{3}$ and $t_{2}=\sqrt{3}-1$. The system of nonlinear equations for $\xi$ and $\eta$

$$
f_{i}(\xi, \eta ; \varphi):=\psi_{3,0}\left(t_{i}\right)=0, \quad i=1,2,
$$


now follows from (18). Some further computations reveal that the equations (20) actually represent two ellipses. More precisely, (20) is equivalent to

$$
e_{i}(\xi, \eta ; \varphi)=\frac{\left(\xi-p_{i}(\varphi)\right)^{2}}{a_{i}^{2}}+\frac{\left(\eta-q_{i}(\varphi)\right)^{2}}{b_{i}^{2}}-1=0, \quad i=1,2,
$$

where the coordinates of the centres of the ellipses are

$$
\begin{aligned}
& \left(p_{1}(\varphi), q_{1}(\varphi)\right)=\left(\frac{1}{9}(3-4 \sqrt{3}) \cos \varphi, \frac{1}{9}(-3-4 \sqrt{3}) \sin \varphi\right) \\
& \left(p_{2}(\varphi), q_{2}(\varphi)\right)=\left(\frac{1}{9}(-3-8 \sqrt{3}) \cos \varphi, \frac{1}{9}(-9-8 \sqrt{3}) \sin \varphi\right)
\end{aligned}
$$

and the semiaxes are

$$
\begin{aligned}
& \left(a_{1}, b_{1}\right)=\left(\frac{2}{9}(3+2 \sqrt{3}), \frac{2}{9}(12+7 \sqrt{3})\right), \\
& \left(a_{2}, b_{2}\right)=\left(\frac{4}{9}(3+2 \sqrt{3}), \frac{2}{9}(9+5 \sqrt{3})\right) .
\end{aligned}
$$

Thus the solution of the system of nonlinear equations (20) is given by the intersection of two ellipses (21). Therefore, it is enough to show that this two particular ellipses intersect in $\mathcal{D}_{3,0}:=\{(\xi, \eta) ; \xi>1, \eta>0\}$. An example of such ellipses for $\varphi=\pi / 4$ and $\varphi=\pi / 2$ is shown on Fig. 1. It is clearly seen that there is precisely one intersection in $\mathcal{D}_{3,0}$. This will now be confirmed by the following lemma.

Lemma 4. The ellipses $e_{i}(\xi, \eta ; \varphi)=0, i=1,2$, intersect precisely at one point in $\mathcal{D}_{3,0}$.

Proof. The prove will base on the fact which is depicted in Fig. 1 in the shadowed region. We shall prove that similar situation appears for each $\varphi \in$ $(0, \pi / 2]$. A closer look to ellipses $e_{i}=0, i=1,2$, reveals that they actually rotate when $\varphi$ runs over the interval $(0, \pi / 2]$. It can also be shown that their centres rotate along two other ellipses which are uniquely determined by $(22)$ and (23). Let $\xi_{i}$ be the solutions of $e_{i}(\xi, 0 ; \varphi)=0$ on the boundary of $\mathcal{D}_{3,0}$, $i=1,2$, respectively. Similarly, let $\eta_{i}$ be the solutions of $e_{i}(1, \eta ; \varphi)=0$ on 


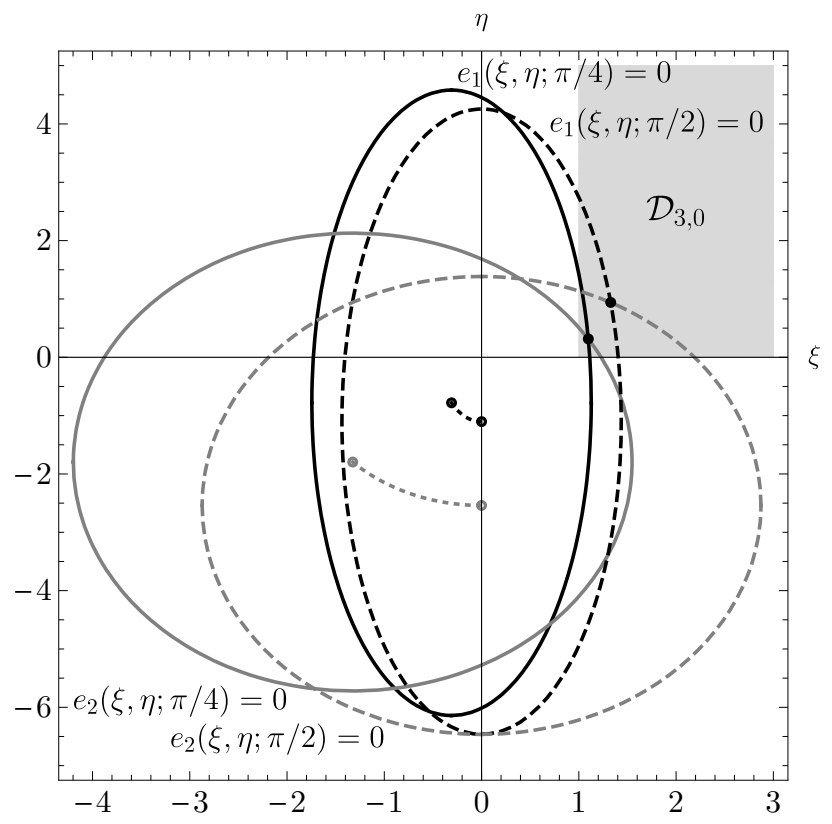

Figure 1: Graphs of ellipses $e_{1}(\xi, \eta ; \pi / 4)=0$ (solid black), $e_{1}(\xi, \eta ; \pi / 2)=0$ (dashed black), $e_{2}(\xi, \eta ; \pi / 4)=0$ (solid gray) and $e_{2}(\xi, \eta ; \pi / 2)=0$ (dashed gray). In the middle of the figure are loci along which centres of ellipses are moving for $\varphi \in[\pi / 4, \pi / 2]$. The region $\mathcal{D}_{3,0}$ is shadowed upper right. Solutions of the nonlinear system (21) for $\varphi=\pi / 4$ and $\varphi=\pi / 2$ are black points in $\mathcal{D}_{3,0}$.

the boundary of $\mathcal{D}_{3,0}, i=1,2$, respectively. To prove that there is precisely one intersection point in $\mathcal{D}_{3,0}$, it is enough to see that

$$
\left(\xi_{1}-\xi_{2}\right)\left(\eta_{1}-\eta_{2}\right)<0 .
$$

Since $e_{i}(\xi, 0 ; \varphi)=0, i=1,2$, are quadratic equations in $\xi$, the intersections $\xi_{i}, i=1,2$, are easily determined. It turns out that

$$
\begin{aligned}
& \xi_{1}=\frac{1}{9}(3-4 \sqrt{3}) \cos \varphi+\sqrt{\frac{1}{54}(19+52 \sqrt{3})+\frac{1}{54}(37-20 \sqrt{3}) \cos 2 \varphi}, \\
& \xi_{2}=-\frac{1}{9}(3+8 \sqrt{3}) \cos \varphi+\sqrt{\frac{1}{27}(74+59 \sqrt{3})+\frac{1}{27}(38+5 \sqrt{3}) \cos 2 \varphi .}
\end{aligned}
$$


It is similarly easy to see that the solutions of $e_{i}(1, \eta ; \varphi)=0, i=1,2$, are

$$
\begin{aligned}
\eta_{1} & =-\frac{1}{9}(3+4 \sqrt{3}) \sin \varphi \\
& +\sqrt{\frac{2}{27}(199+116 \sqrt{3})+\frac{2}{27}(37+20 \sqrt{3}) \cos \varphi} \sin \frac{\varphi}{2}, \\
\eta_{2} & =-\frac{1}{9}(9+8 \sqrt{3}) \sin \varphi \\
& +\sqrt{\frac{1}{27}(362+213 \sqrt{3})+\frac{1}{27}(182+99 \sqrt{3}) \cos \varphi} \sin \frac{\varphi}{2} .
\end{aligned}
$$

A straightforward computation using some basic properties of trigonometric functions leads to (24).

Thus we can state the following theorem.

Theorem 5. The system of nonlinear equations (20) has a unique admissible solution $\left(\xi^{*}, \eta^{*}\right)$ in $\mathcal{D}_{3,0}$. Consequently, there exists the unique optimal cubic $G^{0}$ approximant of the circular arc, given by the central angle $2 \varphi \in(0, \pi]$. The error of the approximation is

$$
\max _{t \in[-1,1]}\left|\psi_{3,0}^{*}(t)\right|=\frac{1}{64}(26-15 \sqrt{3}) \varphi^{6}+\mathcal{O}\left(\varphi^{8}\right) \approx 0.0003 \varphi^{6}+\mathcal{O}\left(\varphi^{8}\right) .
$$

Proof. The existence and the uniqueness of the optimal solution follows from the previous lemma. The asymptotic expansion can be obtained from the Taylor expansion of $\psi_{3,0}^{*}(0)$ around $\varphi=0$ considering analytic solution for $\xi^{*}$ and $\eta^{*}$ (rather longish formulae which will not be written here) and using some computer algebra system.

Although the exact formulae for $\xi^{*}$ and $\eta^{*}$ from Theorem 5 can, in principal, be obtained, they will probably be evaluated numerically in practice. One can use a particular iterative method (e.g., Newthon-Raphson method), since quite accurate starting points for the iteration can be obtained by finding an approximate intersection of the ellipses in $\mathcal{D}_{3,0}$. For $\varphi=\pi / 2$, the optimal solution becomes particularly simple, namely $\xi^{*}=4 \sqrt{2+4 \sqrt{3}} / 9$ and $\eta^{*}=$ $(5+2 \sqrt{3}) / 9$. Fig. 2 shows the approximant together with the error in this case. As a comparison, we took the approximant arising from the choice of $p_{6,0}$ having uniformly distributed zeros on $[-1,1]$, i.e., $\pm 1, \pm 1 / 5$ and $\pm 3 / 5$. The corresponding error is also shown on Fig. 2 and it is much bigger. 

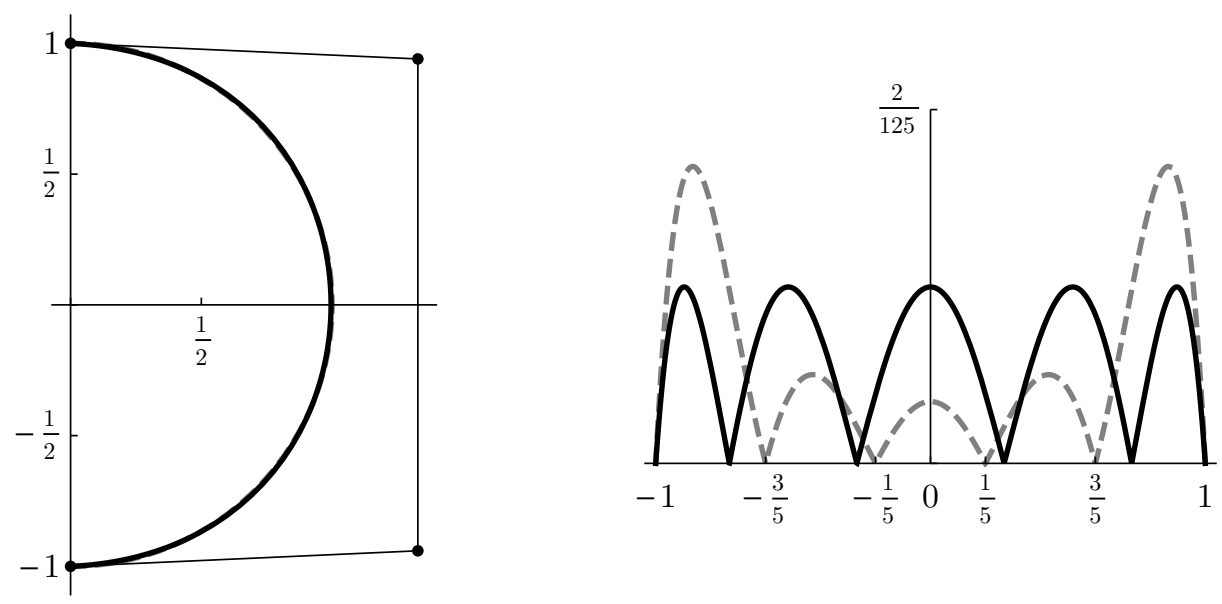

Figure 2: Left: The semicircle (dashed gray, invisible) together with the optimal cubic $G^{0}$ approximant (black). Right: Graphs of errors $\left|\psi_{3,0}^{*}\right|$ (solid black) and $\left|\psi_{3,0}\right|$ possessing equidistant zeros (dashed gray).

The $G^{1}$ approximation by cubics was considered in [14] and we will skip it here. Our approach relying on Subsection 3.3 confirms the results.

Finally, let us consider the quartic $G^{1}$ case for which the optimality of the solution has not been studied yet. The control points of the parametric polynomial approximant $\boldsymbol{p}_{4}$ are

$$
\begin{aligned}
& \boldsymbol{b}_{0}=(\cos \varphi,-\sin \varphi)^{T}, \quad \boldsymbol{b}_{1}=\boldsymbol{b}_{0}+d(\sin \varphi, \cos \varphi)^{T}, \quad \boldsymbol{b}_{2}=(\xi, 0)^{T}, \\
& \boldsymbol{b}_{4}=(\cos \varphi, \sin \varphi)^{T}, \quad \boldsymbol{b}_{3}=\boldsymbol{b}_{4}-d(-\sin \varphi, \cos \varphi)^{T},
\end{aligned}
$$

where $d>0$. The approximants again form a two-parametric family as it was the case in the $G^{0}$ cubic approximation. But it is expected that the problem is harder to be analyzed and this is probably the reason that there are no results on the optimal solution available in the literature. We will again follow our approach and show that the solution provides the smallest known error.

By (18), we have to find all solutions $(\xi, d)$ of the system

$$
\psi_{4,1}\left(t_{i}\right)=0, \quad i=1,2,
$$

where $0<t_{1}<t_{2}<1$ are zeros of $p_{8,1}^{*}$ given by (15) in Subsection 3.2. 
First note that by $(17), E(t):=\psi_{4,1}(t)=0$ is an ellipse in the coordinate system $(\xi, d)$ for any fixed $t \in(0,1)$. It can be written as

$$
\begin{aligned}
\left(4 d\left(1-t^{4}\right) \sin \varphi+\right. & \left.3 \xi\left(1-t^{2}\right)^{2}+\left(5+6 t^{2}-3 t^{4}\right) \cos \varphi\right)^{2} \\
& +16 t^{2}\left(2 d\left(1-t^{2}\right) \cos \varphi-\left(3-t^{2}\right) \sin \varphi\right)^{2}=64 .
\end{aligned}
$$

The following lemma will be crucial for determining the locations of the solutions of (25).

Lemma 6. The ellipses $E\left(u_{1}\right)=0$ and $E\left(u_{2}\right)=0$ intersect in precisely four points for any pair $u_{1}, u_{2} \in(0,1), u_{1} \neq u_{2}$.

Proof. A detailed proof requires several technical calculations. We will omit some details here and present the most important observations. The idea is the following. It is not difficult to see that the points $(0,0)$ and $\left(0, \frac{1}{2} \sin \varphi\right)$ are inside the ellipse $E(t)=0$ for all $t \in(0,1)$. Thus, if we choose the half lines $L_{1}:\{0\} \times(-\infty, 0], L_{2}:(-\infty, 0] \times\{0\}, L_{3}:\{0\} \times[0, \infty)$, $L_{4}:[0, \infty) \times\left\{\frac{1}{2} \sin \varphi\right\}$, they will intersect each ellipse $E(t)=0$ precisely once (see Fig. 3). If we can prove that as $t$ is increasing the point $E(t) \cap$ $L_{i}$ is moving towards the origin for vertical half lines, i.e., for $i=1,3$, and it is moving away from the origin for horizontal half lines, i.e., $i=$ 2,4 , similar argument as (24) ensures that for any pair $u_{1}, u_{2} \in(0,1)$ one has at least one intersection of $E\left(u_{1}\right)=0$ and $E\left(u_{2}\right)=0$ in each of the sets $\mathcal{Q}_{0}=\{(\xi, d) ; \xi<0, d<0\}, \mathcal{Q}_{1}=\left\{(\xi, d) ; \xi>0, d<\frac{1}{2} \sin \varphi\right\}, \mathcal{Q}_{2}=$ $\left\{(\xi, d) ; \xi>0, d>\frac{1}{2} \sin \varphi\right\}$ and $\mathcal{Q}_{3}=\{(\xi, d) ; \xi<0, d>0\}$.

Here we give a sketch of the proof without detailed technical computations. The main argument we use for all four different cases is the following. If $f$ is an arbitrary function on $[0,1]$, then $f \geq 0$ on $(0,1)$, if $f(0) \geq 0$ and $f^{\prime} \geq 0$ on $(0,1)$ or $f(1) \geq 0$ and $f^{\prime} \leq 0$ on $(0,1)$.

Let $F_{i}(t)$ be the nonconstant component of the intersection $E(t) \cap L_{i}$, $i=1,2,3,4$. Clearly, $F_{i}(t)$ is the solution of a quadratic equation (26), hence it is of the form

$$
F_{i}(t)=\alpha_{i}(t)^{-1}\left(\beta_{i}(t)+\sqrt{\gamma_{i}(t)}\right),
$$

with $\alpha_{i}, \beta_{i}$ and $\gamma_{i}$ being some polynomials. Our goal is to prove that $F_{i}$ is a monotone function on $(0,1)$. If we define $f_{i, 1}(t):=\alpha_{i}(t) \beta_{i}^{\prime}(t)-\alpha_{i}^{\prime}(t) \beta_{i}(t)$ and $f_{i, 2}(t):=\frac{1}{2} \alpha_{i}(t) \gamma_{i}^{\prime}(t)-\alpha_{i}^{\prime}(t) \gamma_{i}(t)$, then the derivative of $F_{i}$ is of the form

$$
F_{i}^{\prime}(t)=\left(\alpha_{i}^{2}(t) \sqrt{\gamma_{i}(t)}\right)^{-1}\left(f_{i, 1}(t) \sqrt{\gamma(t)}+f_{i, 2}(t)\right) .
$$


The fact that $F_{1}$ is increasing and $F_{2}$ is decreasing now follows from $f_{1,1} \geq 0$, $f_{1,2} \geq 0, f_{2,1} \leq 0$ and $f_{2,2} \leq 0$, and the fact that $F_{3}$ is decreasing and $F_{4}$ is increasing follows from the inequalities $f_{3,1} \geq 0, f_{3,2} \leq 0, f_{4,1} \leq 0, f_{4,2} \geq 0$ and $f_{i, 1}^{2} \gamma_{i} \leq f_{i, 2}^{2}, i=3,4$. Let us only prove formally that $F_{4}$ is increasing. All the other cases can be verified in a similar way.

By (26) and (27) we have

$$
\begin{aligned}
\alpha_{4}(t) & =\frac{3}{8}\left(1-t^{2}\right)^{2} \\
\beta_{4}(t) & =\frac{1}{8}\left(\left(3 \cos \varphi+2 \sin ^{2} \varphi\right) t^{4}-6 \cos \varphi t^{2}+\cos 2 \varphi-5 \cos \varphi-1\right) \\
\gamma_{4}(t) & =-\sin ^{2} \varphi \sin ^{4}\left(\frac{\varphi}{2}\right) t^{6}+4 \cos ^{2}\left(\frac{\varphi}{2}\right) \sin ^{4}\left(\frac{\varphi}{2}\right)(3-\cos \varphi) t^{4} \\
& -\frac{1}{16}(\sin 2 \varphi-6 \sin \varphi)^{2} t^{2}+1
\end{aligned}
$$

SO

$$
\begin{aligned}
f_{4,1}(t) & =\frac{3}{8} t\left(1-t^{2}\right)\left(\sin ^{2} \varphi t^{2}-\sin ^{2} \varphi-4 \cos \varphi\right) \\
f_{4,2}(t) & =-\frac{3}{32} t\left(1-t^{2}\right) \\
& \times\left((1-\cos \varphi)^{2} \sin ^{2} \varphi t^{6}-8 \cos ^{2}\left(\frac{\varphi}{2}\right) \sin ^{4}\left(\frac{\varphi}{2}\right)(9-\cos \varphi) t^{4}\right. \\
& \left.-\sin ^{2} \varphi\left(\cos ^{2} \varphi+2 \cos \varphi-15\right) t^{2}+\sin ^{2} \varphi(3-\cos \varphi)^{2}-16\right) .
\end{aligned}
$$

If we write $s=t^{2}$, then $f_{4,1}(\sqrt{s})=\frac{3}{8} \sqrt{s}(1-s) \widetilde{f}_{4,1}(s)$, where $\widetilde{f}_{4,1}(s)=$ $\sin ^{2} \varphi s-4 \cos \varphi-\sin ^{2} \varphi$. Obviously $\widetilde{f}_{4,1}(1)=-4 \cos \varphi \leq 0$ and $\widetilde{f}_{4,1}^{\prime}(s)=$ $\sin ^{2} \varphi>0$ on $(0,1)$. Thus $\widetilde{f}_{4,1} \leq 0$ and consequently $f_{4,1} \leq 0$ on $(0,1)$. Similarly, $f_{4,2}(\sqrt{s})=-\frac{3}{32} \sqrt{s}(1-s) \widetilde{f}_{4,2}(s)$, where $\widetilde{f}_{4,2}(1)=-16 \cos ^{2} \varphi \leq 0$ and $\widetilde{f}_{4,2}^{\prime}(s)=\sin \varphi \widetilde{g}_{4,2}(s)$. Moreover, $\widetilde{g}_{4,2}(1)=6 \sin 2 \varphi \geq 0$ and $\widetilde{g}_{4,2}^{\prime}(s)=$ $-8 \cos \left(\frac{\varphi}{2}\right) \sin ^{3}\left(\frac{\varphi}{2}\right) \widetilde{h}_{4,2}(s)$, where $\widetilde{h}_{4,2}(1)=2(3+\cos \varphi)>0$ and $\widetilde{h}_{4,2}^{\prime}(s)=$ $-3(1-\cos \varphi)<0$. This implies $\widetilde{h}_{4,2}>0, \widetilde{g}_{4,2} \geq 0$ and $\widetilde{f}_{4,2} \leq 0$, thus $f_{4,2} \geq 0$ on $(0,1)$.

It remains to prove that $k_{4}:=f_{4,1}^{2} \gamma_{4}-f_{4,2}^{2} \leq 0$. If we write $s=t^{2}$, then

$$
k_{4}(\sqrt{s})=-\frac{9}{512} s(1-s)^{5} \cos ^{2}\left(\frac{\varphi}{2}\right) \sin ^{6}\left(\frac{\varphi}{2}\right) \widetilde{k}_{4}(s),
$$

and it is enough to prove that $\widetilde{k}_{4} \geq 0$ on $(0,1)$. A computer algebra system 
helps us to verify that

$$
\begin{aligned}
\widetilde{k}_{4}^{\prime}(s) & =-4 \sin ^{2} \varphi\left(24 \sin ^{4}\left(\frac{\varphi}{2}\right) s^{2}-(6 \cos 2 \varphi-56 \cos \varphi+50) s\right. \\
& +3 \cos 2 \varphi-44 \cos \varphi+89),
\end{aligned}
$$

and

$$
\max _{s \in \mathbb{R}} \widetilde{k}_{4}^{\prime}(s)=-\frac{64}{3} \sin ^{2} \varphi<0
$$

Since $\widetilde{k}_{4}(1)=256 \cos \varphi \geq 0$, we have $\widetilde{k}_{4} \geq 0$. Thus, by $(28), k_{4} \leq 0$ on $(0,1)$ and the proof is completed.
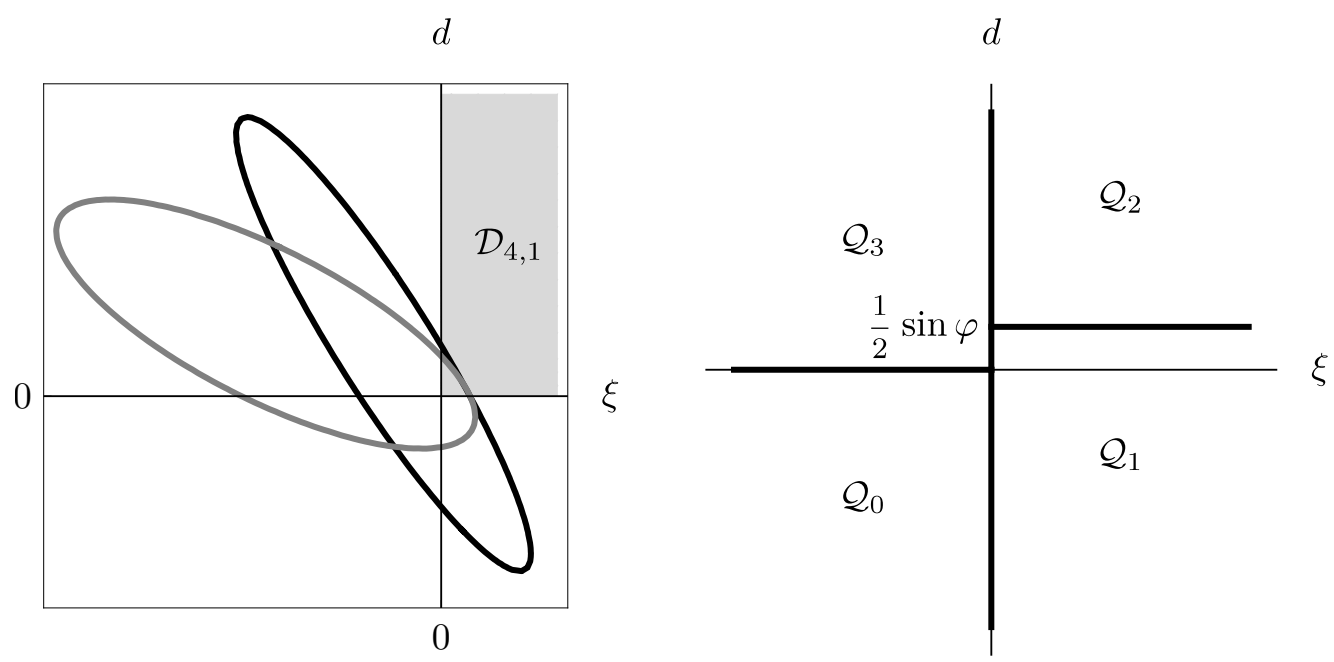

Figure 3: Left: Graphs of the ellipses $E\left(u_{i}\right)=0, i=1,2$. The upper right corner is the admissible region for the intersections. Right: The four regions $\mathcal{Q}_{i}, i=0,1,2,3$, each with precisely one solution of (25).

It follows from the previous lemma that at least one solution exists in each of the sets $\mathcal{Q}_{i}, i=0,1,2,3$. Since two ellipses can not intersect in more than four points, there are no additional solutions. However, solutions in $\mathcal{Q}_{0}$ are not admissible, since $d<0$. If the solution is in $\mathcal{Q}_{3}$, then $\xi<0$ and an analysis of $\boldsymbol{p}_{4}(0)$ reveals that its first coordinate is negative, which is clearly not admissible. Thus it remains to study the solutions in $\mathcal{Q}_{i}, i=1,2$. The solution in $\mathcal{Q}_{2}$ is always admissible, since $\xi, d>0$. It may happen that there 
is another admissible solution in $\mathcal{Q}_{1}$. As it will turn out, this depends on the parameter $\varphi$. In order to better understand a general situation, let us define the Gröbner basis $\mathcal{G}$ of $E\left(u_{1}\right)$ and $E\left(u_{2}\right), u_{1}<u_{2}$, according to the lexicographic ordering $\xi \succ d$. Furthermore, let $g \in \mathcal{G} \cap \mathbb{R}[d]$. The following lemma characterizes its zeros.

Lemma 7. The polynomial $g$ is a quartic with four real zeros $d_{0}<d_{1}<$ $d_{2}<d_{3}$, where $d_{0}<0$ and $d_{1}<\frac{1}{2} \sin \varphi<d_{2}$. Let

$$
\begin{aligned}
& \tilde{\varphi}\left(u_{1}, u_{2}\right) \\
& =\frac{1}{2} \arccos \left(\frac{u_{1}^{4}\left(u_{2}^{2}-4\right)^{2}-u_{1}^{2}\left(8 u_{2}^{4}-46 u_{2}^{2}+40\right)+\left(4 u_{2}^{2}-9\right)^{2}+32 u_{2}^{2}-64}{u_{1}^{4}\left(u_{2}^{2}-4\right)^{2}-u_{1}^{2}\left(8 u_{2}^{4}-46 u_{2}^{2}+40\right)+\left(4 u_{2}^{2}-9\right)^{2}-32 u_{1}^{2}}\right) .
\end{aligned}
$$

If $0<\varphi \leq \widetilde{\varphi}\left(u_{1}, u_{2}\right)$ then $d_{1} \geq 0$, otherwise $d_{1}<0$.

PROOF. Some computer algebra system reveals that $g(d)=\sum_{j=0}^{4} \delta_{j} d^{j}$, with

$$
\begin{aligned}
\delta_{0} & =\frac{1}{2}\left(u_{1}^{2}-1\right)^{4}\left(u_{2}^{2}-1\right)^{4}\left(u_{2}^{2}-u_{1}^{2}\right) \sin ^{2} \varphi \\
& \times\left(\left(u_{1}^{4}\left(u_{2}^{2}-4\right)^{2}-u_{1}^{2}\left(8 u_{2}^{4}-46 u_{2}^{2}+40\right)+\left(4 u_{2}^{2}-9\right)^{2}-32 u_{1}^{2}\right) \cos 2 \varphi\right. \\
& \left.-u_{1}^{4}\left(u_{2}^{2}-4\right)^{2}+u_{1}^{2}\left(8 u_{2}^{4}-46 u_{2}^{2}+40\right)-\left(4 u_{2}^{2}-9\right)^{2}-32 u_{2}^{2}+64\right), \\
\delta_{4} & =2\left(u_{1}^{2}-1\right)^{4}\left(u_{2}^{2}-1\right)^{4}\left(u_{1}^{2}-u_{2}^{2}\right) \\
& \times\left(\left(u_{1}^{2}-1\right)^{2}\left(u_{2}^{2}-1\right)^{2} \cos 4 \varphi+4\left(u_{1}^{4}-1\right)\left(u_{2}^{4}-1\right) \cos 2 \varphi\right. \\
& \left.+3\left(u_{1}^{4}+u_{1}^{4} u_{2}^{4}+u_{2}^{4}+1\right)+2\left(u_{1}^{4} u_{2}^{2}+u_{1}^{2} u_{2}^{4}-10 u_{1}^{2} u_{2}^{2}+u_{1}^{2}+u_{2}^{2}\right)\right) .
\end{aligned}
$$

Recall that $u_{1}<u_{2}$. A rather technical analysis of $\delta_{4}$ implies that $\delta_{4}<0$. From Lemma 6 it follows that $g$ has four real zeros $d_{0}<d_{1}<d_{2}<d_{3}$, for which $d_{0}<0, d_{1}<\frac{1}{2} \sin \varphi<d_{2}$ (see Fig. 4). Since $\delta_{4} \prod_{i=0}^{3} d_{i}=\delta_{0}$, it follows that $d_{1}$ and $\delta_{0}$ must be of the same sign. Thus we have to study the equation $\delta_{0}=g(0)=0$ with respect to $\varphi$. A straightforward calculations lead to the unique solution on $(0, \pi / 2)$ given by $(29)$. Some further calculations also show that $\delta_{0} \geq 0$ for $\varphi \leq \widetilde{\varphi}\left(u_{1}, u_{2}\right)$, and the result of the lemma follows.

It is clear from the previous lemma that our problem of the optimal $G^{1}$ quartic approximation has at most two admissible solutions. For $\varphi \geq$ $\widetilde{\varphi}\left(u_{1}, u_{2}\right)$ the solution is uniquely determined by $d_{2}$ and the corresponding 

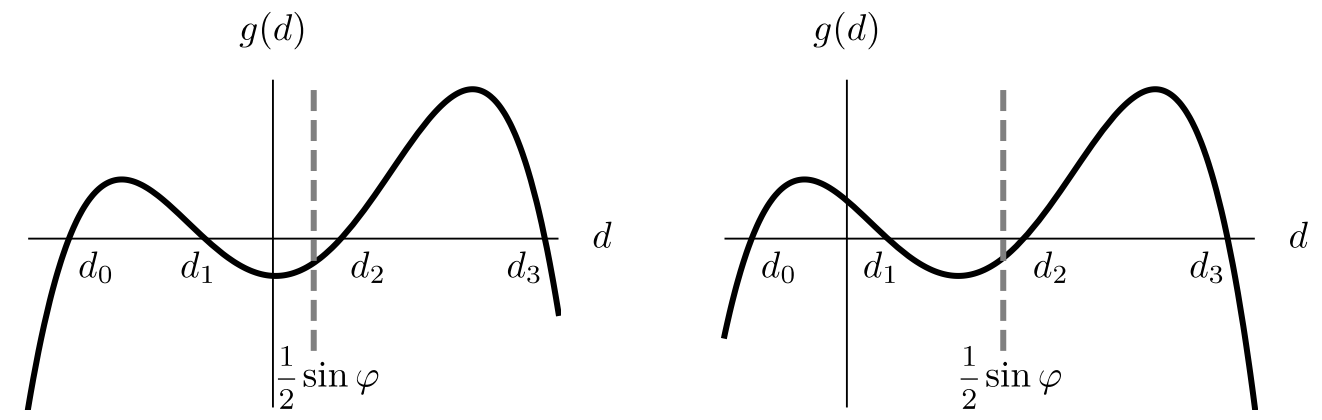

Figure 4: Two characteristic graphs of $g$ (solid black) with locations of zeros $d_{i}, i=$ $0, \ldots, 3$, and vertical lines $d=\frac{1}{2} \sin \varphi$ (dashed gray).

$\xi_{2}$. If $\varphi<\widetilde{\varphi}\left(t_{1}, t_{2}\right)$, Lemma 7 ensures the existence of an another admissible solution arising from $d_{1}>0$. We shall see now that this solution is inferior comparing to the solution given by $d_{2}$ if the simplified radial error is considered.

Lemma 8. If $\varphi<\widetilde{\varphi}\left(u_{1}, u_{2}\right)$, the asymptotic expansions of $\psi_{4,1}(0)$ for the solutions given by $\left(\xi_{i}, d_{i}\right), i=1,2$, are

$$
\frac{1}{64}(17+12 \sqrt{12}) u_{1} u_{2} \varphi^{8}+\mathcal{O}\left(\varphi^{9}\right), \quad \frac{1}{64}(17-12 \sqrt{12}) u_{1} u_{2} \varphi^{8}+\mathcal{O}\left(\varphi^{9}\right) .
$$

Proof. Since the zeros of $g$ smoothly depend on $\varphi$, we can assume that each of them can be written as $d(\varphi)=\sum_{j=0}^{\infty} c_{j} \varphi^{j}$. In order to compute the coefficients $c_{j}, j=0,1,2, \ldots$, we consider the equation

$$
g(d(\varphi))=g\left(\sum_{j=0}^{\infty} c_{j} \varphi^{j}\right)=0 .
$$

With the help of computer algebra system we can derive a system of linear equations for $c_{j}, j=0,1, \ldots, 8$. The solutions correspond to the expansions of four solutions $g(d)=0$. By Lemma 7, $0<d_{1}<\frac{1}{2} \sin \varphi<d_{2}$, and it is easy to choose the right solution for each of $d_{i}, i=1,2$. These expansions are further used to obtain appropriate expansions of $\xi_{i}(\varphi), i=1,2$, and finally for the expansions of $\psi_{4,1}(0)$.

We are now ready to state the main theorem concerning the optimal $G^{1}$ quartic approximation of the circular arc $\boldsymbol{c}$ given by an inner angle $2 \varphi$. Since 
we are interested in the optimal solution, we have to choose $u_{1}=t_{1}$ and $u_{2}=t_{2}$. In this case the simplified radial error $\psi_{4,1}^{*}$ attains its maximum at $t=0$ and previous lemmas imply the following result.

Theorem 9. If $\varphi \geq \widetilde{\varphi}$, where $\widetilde{\varphi}=\widetilde{\varphi}\left(t_{1}, t_{2}\right) \approx 0.76$ is given by (29), then there exists the unique admissible $G^{1}$ quartic approximant of the circular arc $\boldsymbol{c}$, given by the solution $\left(\xi_{2}, d_{2}\right)$. For $\varphi<\widetilde{\varphi}$, there are two admissible approximants arising from $\left(\xi_{i}, d_{i}\right), i=1,2,0<d_{1}<d_{2}$. Their asymptotic expansions of the simplified radial error, i.e., $\max _{t \in[-1,1]}\left|\psi_{4,1}^{*}(t)\right|=\left|\psi_{4,1}^{*}(0)\right|$, are

$$
\begin{aligned}
& \frac{1}{64}(17+12 \sqrt{12}) t_{1} t_{2} \varphi^{8}+\mathcal{O}\left(\varphi^{9}\right) \approx 1.0 \times 10^{-2} \varphi^{8}+\mathcal{O}\left(\varphi^{9}\right), \\
& \frac{1}{64}(17-12 \sqrt{12}) t_{1} t_{2} \varphi^{8}+\mathcal{O}\left(\varphi^{9}\right) \approx 8.7 \times 10^{-8} \varphi^{8}+\mathcal{O}\left(\varphi^{9}\right),
\end{aligned}
$$

respectively.

This theorem provides a full characterization of optimal $G^{1}$ quartic approximants of the circular arc $\boldsymbol{c}$. It can be additionally shown that their second components are monotone functions, thus they are also regular curves, but we will skip the poof of this fact.

Let us provide some numerical examples confirming the theoretical results. As the first one, the approximation of the unit semicircle will be considered. In this case $\varphi=\pi / 2$ and by Theorem 9 there exists the unique admissible optimal $G^{1}$ quartic approximant. It is shown on Fig. 5. Numerical values for the parameters are $\xi_{2} \approx 1.5051$ and $d_{2} \approx 0.8715$. These values can be obtained also in a closed form, but the expressions are to long to be presented here.

As the second example let us consider $\varphi=\pi / 5$. Since $\varphi<\widetilde{\varphi}\left(t_{1}, t_{2}\right)$, Theorem 9 implies the existence of two admissible approximants. The numerical values for the parameters are $\xi_{1} \approx 1.2292, d_{1} \approx 0.1141$ and $\xi_{2} \approx 1.0681$, $d_{2} \approx 0.3192$. Theorem 9 further asserts that the second approximant provides a better approximation which is clearly seen on Fig. 6.

Finally, let us compare our proposed approximant by some known approximants from the literature. In Table 1 Hausdorff distances of the known quartic $G^{1}$ approximants and the circular arc given by the inner angle $\pi / 2$ $(\varphi=\pi / 4)$ are collected. Our solution clearly provides the smallest error.

It is also possible to study $G^{2}$ quartic approximation using our approach and the results from Subsection 3.3. The family of geometric approximants 

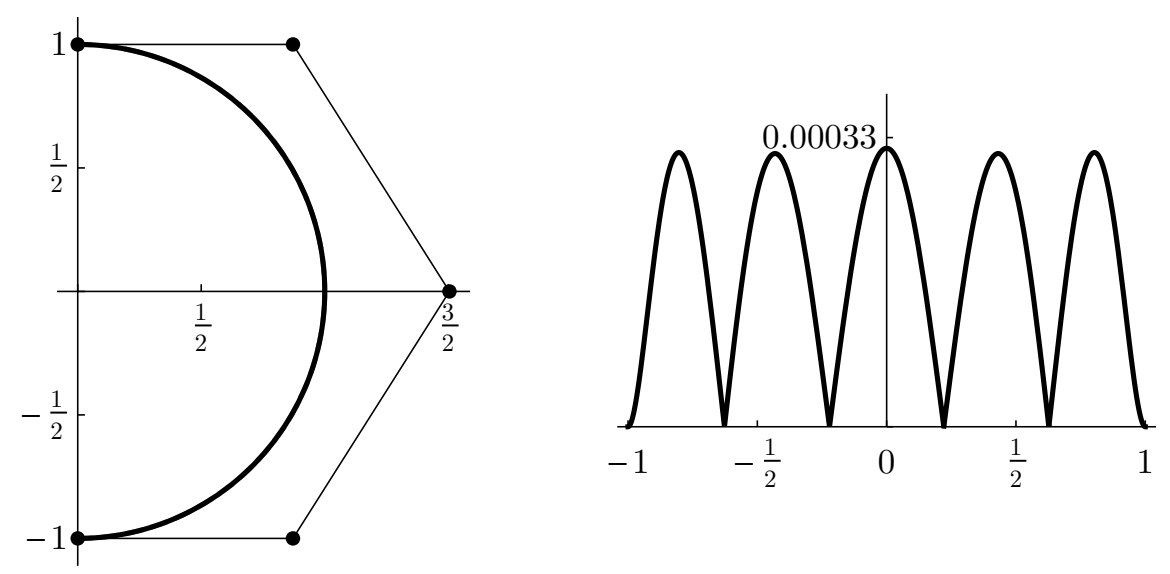

Figure 5: Left: The semicircle (dashed gray, invisible) together with the optimal $G^{1}$ quartic approximant (black). Right: The graph of the error $\left|\psi_{4,1}^{*}\right|$.

\begin{tabular}{|c|r|c|}
\hline Zeros of $p_{81}$ & Hausdorff distance & Reference \\
\hline$-1,-1,-1,-1,1,1,1,1$ & $3.50 \times 10^{-5}$ & {$[3]$} \\
\hline$-1,-1,-w_{1},-w_{1}, w_{1}, w_{1}, 1,1$ & $4.75 \times 10^{-6}$ & {$[13]$} \\
\hline$-1,-1,-1,0,0,1,1,1$ & $3.55 \times 10^{-6}$ & {$[3]$} \\
\hline$-1,-1,0,0,0,0,1,1$ & $2.03 \times 10^{-6}$ & {$[22]$} \\
\hline$-1,-1,-1 / 2,0,0,1 / 2,1,1$ & $1.11 \times 10^{-6}$ & {$[11]$} \\
\hline$-1,-1,-3 / 5,0,0,3 / 5,1,1$ & $1.08 \times 10^{-6}$ & {$[10]$} \\
\hline$-1,-1,-z_{1}, 0,0, z_{1}, 1,1$ & $7.60 \times 10^{-7}$ & {$[12]$} \\
\hline$-1,-1,-t_{2},-t_{1}, t_{1}, t_{2}, 1,1$ & $6.34 \times 10^{-7}$ & This paper. \\
\hline
\end{tabular}

Table 1: Hausdorff distances of known quartic $G^{1}$ approximants to the circular arc given by inner angle of $\pi / 2$. Zeros in the table are: $w_{1}=\sqrt{2}-1, z_{1}=\frac{1}{3} \sqrt{6-4 \sqrt{3}+2 \sqrt{6} \sqrt{\sqrt{3}-1}}$ and $0<t_{1}<t_{2}<1$ are zeros of $p_{8,1}^{*}$.

depends again on just one parameter and the analysis simplifies significantly comparing to [14]. Only one scalar nonlinear equation has to be analysed with the same solution as it was obtained in [14].

\section{Conclusion}

We have presented a new approach to the solution of the optimal geometric approximation of the circular arc by parametric polynomial curves. 

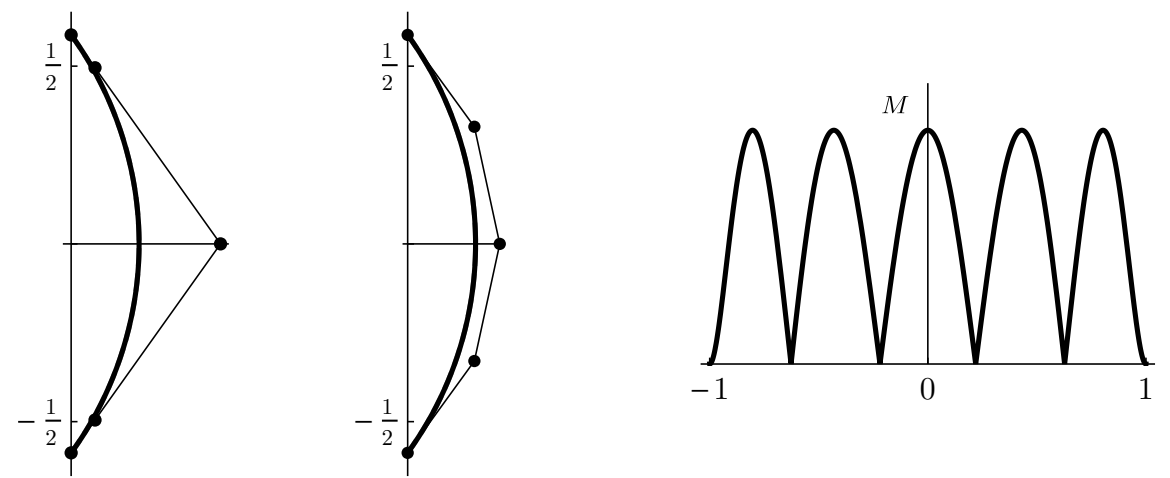

Figure 6: Two $G^{1}$ quartic approximants of the circular arc given by the central angle of $2 \pi / 5$ (dashed gray, invisible). The left one is arising from $d_{1}$, the middle one from $d_{2}$. The error $\left|\psi_{41}^{*}\right|$ (right) is of the same shape for both of them, however the maximum is $M \approx 2.9 \times 10^{-4}$ for the left one and $M \approx 2.1 \times 10^{-7}$ for the middle one.

It is based on the constrained uniform approximation by polynomials. The solutions obtained by the proposed approach coincide with known optimal approximants for $G^{0}$ quadratic, $G^{1}$ cubic and $G^{2}$ quartic case. A general conjecture on the optimality of the geometric approximants was stated and confirmed by a detailed study of the $G^{0}$ cubic and the $G^{1}$ quartic case. As a future work the proposed approach can be used for some other low degree geometric approximants, but some higher order algebraic equations as (11) or (16) have to be solved first. It is not to be expected that the solutions can be given in radicals and numerical procedures are unavoidable. A particularly interesting case would be the $G^{2}$ quintic approximation. But even more important issue would be the proof of Conjecture 3. This would assure that our approach gives the best possible approximants according to the simplified radial distance measure. However, this does not solve the problem of optimality in the sense of Hausdorff distance. To do this, one has to consider the radial error $\widetilde{\psi}$ as a measure of an error. Numerical experiments indicate that this is much more difficult problem.

As an another direction of the future work one could also use similar approach for the approximation of segments of spheres or some other elementary surfaces.

Acknowledgments. The first author acknowledges the financial support from 
the Slovenian Research Agency (ARRS) by research core funding no. P10292, J1-7025, J1-8131. The second author was supported in part by the program P1-0288 and the grant J1-7256 from ARRS.

\section{References}

[1] F. R. Deutsch, P. H. Maserick, Applications of the Hahn-Banach theorem in approximation theory, SIAM Rev. 9 (1967) 516-530.

[2] M. H. Stone, The generalized Weierstrass approximation theorem, Math. Mag. 21 (1948) 167-184, 237-254.

[3] Y. J. Ahn, H. O. Kim, Approximation of circular arcs by Bézier curves, J. Comput. Appl. Math. 81 (1) (1997) 145-163.

[4] G. Jaklič, J. Kozak, On parametric polynomial circle approximation, accepted for publication in Numer. Algorithms.

[5] K. Mørken, Best approximation of circle segments by quadratic Bézier curves, in: Curves and surfaces (Chamonix-Mont-Blanc, 1990), Academic Press, Boston, MA, 1991, pp. 331-336.

[6] T. Dokken, M. Dæhlen, T. Lyche, K. Mørken, Good approximation of circles by curvature-continuous Bézier curves, Comput. Aided Geom. Design 7 (1-4) (1990) 33-41, Curves and surfaces in CAGD '89 (Oberwolfach, 1989).

[7] M. Goldapp, Approximation of circular arcs by cubic polynomials, Comput. Aided Geom. Design 8 (3) (1991) 227-238.

[8] L. Fang, Circular arc approximation by quintic polynomial curves, Comput. Aided Geom. Design 15 (8) (1998) 843-861.

[9] S.-H. Kim, Y. J. Ahn, An approximation of circular arcs by quartic Bézier curves, Comput. Aided Design 39 (6) (2007) 490-493.

[10] Z. Xiaoming, C. Licai, Approximation of circular arcs by quartic Bézier curves, J. Comp.-Aided Design Comp. Graph. 22 (7) (2010) 1094.

[11] Z. Liu, J.-q. Tan, X.-y. Chen, L. Zhang, An approximation method to circular arcs, Appl. Math. Comput. 219 (3) (2012) 1306-1311. 
[12] S. W. Kim, Y. J. Ahn, Circle approximation by quartic $G^{2}$ spline using alternation of error function, J. KSIAM 17 (3) (2013) 171-179.

[13] B. Kovač, E. Žagar, Some new $G^{1}$ quartic parametric approximants of circular arcs, Appl. Math. Comput. 239 (2014) 254-264.

[14] S. Hur, T. Kim, The best $G^{1}$ cubic and $G^{2}$ quartic bézier approximations of circular arcs, J. Comput. Appl. Math. 236 (6) (2011) 1183-1192.

[15] T. Lyche, K. Mørken, A metric for parametric approximation, in: Curves and surfaces in geometric design (Chamonix-Mont-Blanc, 1993), A K Peters, Wellesley, MA, 1994, pp. 311-318.

[16] G. Jaklič, J. Kozak, M. Krajnc, E. Žagar, On geometric interpolation of circle-like curves, Comput. Aided Geom. Design 24 (5) (2007) 241-251.

[17] G. Jaklič, J. Kozak, M. Krajnc, V. Vitrih, E. Žagar, High-order parametric polynomial approximation of conic sections, Constr. Approx. 38 (1) (2013) 1-18.

[18] G. Jaklič, Uniform approximation of a circle by a parametric polynomial curve, Comput. Aided Geom. Design 41 (2016) 36-46.

[19] H. L. Loeb, D. G. Moursund, L. L. Schumaker, G. D. Taylor, Uniform generalized weight function polynomial approximation with interpolation, SIAM J. Numer. Anal. 6 (1969) 284-293.

[20] A. A. Albert, An inductive proof of Descartes' rule of signs, Amer. Math. Monthly 50 (1943) 178-180.

[21] G. Farin, Curves and surfaces for computer-aided geometric design, 4th Edition, Computer Science and Scientific Computing, Academic Press Inc., San Diego, CA, 1997.

[22] S.-H. Kim, Y. J. Ahn, An approximation of circular arcs by quartic Bézier curves, Comput. Aided Design 39 (6) (2007) 490-493. 